\title{
The role of wing veins in colour pattern development in the butterfly Papilio xuthus (Lepidoptera: Papilionidae)
}

\author{
PAUL BERNHARdT KOCH ${ }^{*}$ and H. FrederIK NIJHOUT ${ }^{2}$ \\ ${ }^{1}$ Institute of Zoology I, University of Erlangen-Nürnberg, Staudtstrasse 5, 91058 Erlangen, Germany; e-mail: \\ bkoch@biologie.uni-erlangen.de, bernd.koch@biologie.uni-ulm.de \\ ${ }^{2}$ Department of Biology, Duke University, Durham, NC 27708-0338, USA; e-mail: hfn@acpub.duke.edu
}

Key words. Colour pattern, wing veins, mutants, computer simulation, Papilio xuthus, Lepidoptera

\begin{abstract}
Naturally occurring veinless specimen of the swallowtail Papilio xuthus show an extremely aberrant colour pattern. In spite of the fact that we have no breeding data, these veinless specimen are provisionally called veins-reduced mutant. In these mutants seven longitudinal veins of the fore wing and five of the hind wing are absent. The absence of wing veins is associated with a loss of the broad black venous stripes that normally are present along the proximal portion of the veins. In addition, missing veins cause a loss of the dislocation of black bands in adjacent wing cells, so that what are discrete black segments in normal wings become continuous bands in the veinless wing. Computer simulations show that the morphology of the striped patterns on both the veinless and veined wing can be explained if the wing margin acts as an inductive source of pattern formation and the veins act simply as boundaries to the propagation of the signal from the wing margin. The vein-dependent patterns by contrast, require that the veins act as inductive sources, at least along their proximal portion. This dual role of wing veins is consistent with prior observations on the biology of colour pattern formation. The unique veinless colour pattern strongly supports the hypothesis that the wing margin is the dominant organiser of colour pattern in this species, and possibly in other Papilionidae.
\end{abstract}

\section{INTRODUCTION}

Butterfly wing colour patterns provide a rich source of material for comparative investigations of the principles of pattern development. Early comparative analyses revealed that there is a general underlying principle of colour pattern organisation in butterflies, named the "nymphalid ground plan", because it can be used to explain the structure and diversity of wing patterns in the family Nymphalidae (Schwanwitsch, 1924; Süffert, 1927; Nijhout, 1991). The nymphalid groundplan suggests that wing patterns are composed of three sets of paired pigment bands, the symmetry systems, that run from the anterior to the posterior margin of the wing and called respectively, the basal-, central-, and border-symmetry systems. Strikingly, in many colour patterns these pigment bands typically have discontinuities or dislocations where they cross a wing vein. The reason for these dislocations is that pattern determination in each wing-cell (a wing-cell is the area bounded by wing veins) is partially uncoupled from pattern determination in adjoining wing cells. The wing veins appear to act as barriers to the propagation of determinative signals so that in each wingcell the processes of pattern formation become semiindependent: they obey common genetic rules but are causally uncoupled from each other, much like left and right sides of a bilaterally symmetrical organism are uncoupled from each other.

Relatively little information is presently available on the sites of origin of the morphogenetic signals that organise the colour pattern. By far the best understood mechanism for colour pattern induction is that concerned with the development of eyespots of the Nymphalidae. Transplantation experiments have demonstrated that eyespots are induced by morphogenetic signals that emerge from small clusters of cells, called the foci (Nijhout, 1980; French \& Brakefield, 1992). These organising centres themselves are induced by a complex pattern of expression of the Distal-less gene (Carroll et al., 1994; Brakefield et al., 1996) and the hedgehog signalling pathway (Keys et al., 1999). The signals that emerge from these organising centres determine the size and pigmentation of the various concentric coloured rings that make up an eyespot (Monteiro et al., 1997a,b; Brakefield, 1998).

In addition to the specialised cells that induce eyespots, there is evidence that the wing veins and wing margin play a role in the induction of the pigment patterns in their immediate vicinity. Most species of Nymphalidae have narrow pigment bands that run along the distal wing margin. If the wing margin is surgically cut early in development, then the marginal colour patterns fail to develop wherever the margin is damaged (Nijhout \& Grunert, 1988). Evidence for an active role of wing veins in pattern formation (as opposed to their restrictive role outlined above) is less direct and comes primarily from comparative morphological observations that some colour patterns are formed parallel to the wing veins, and that vein aberrations are often associated with local pattern aberrations (Nijhout, 1991; Brakefield et al., 1996).

In the present study we analyse the dramatic changes in the colour pattern of the veins-reduced mutant of the papilionid Papilio xuthus. Our analyses and computer

\footnotetext{
* Corresponding author.
} 
simulations of colour patterns on normal and veinsreduced wings demonstrate that in this species the wing veins and wing margin play a dominant if not exclusive role in colour pattern formation.

\section{MATERIAL AND METHODS}

A series of male and female specimen of the Japanese Papilio xuthus L. (Papilionidae, Lepidoptera) were collected near the Nagoya Castle in Nagoya, Japan, in 1985 by Dr. Katsuhiko Endo (Yamaguchi University, Japan), who kindly provided the material. These butterflies are missing most of the longitudinal veins on each wing (Fig.1 a, d). The veins of the anterior wing margin and the discal cell appeared normal. Interestingly enough, although many veins are missing, the wings were more or less regularly inflated after adult emergence, and these animals were able to fly reasonably well. Because many nearly identical specimens were collected (about 7 in two collections) it is assumed we are dealing with a genetic mutation. Unfortunately, it has proven impossible to breed these animals and no additional specimen have been caught in following years Although we have not been able to obtain breeding data on these animals, the fact that multiple individuals were collected from a single location at the same time suggest the defect resulted from a mutation within a single brood. The specimens described here are provisionally called veins-reduced mutants.

Computer simulation of the effect of venation on the colour pattern was done as follows. A segment of a wing was represented by a rectangular array of $100 \times 200$ nodes. The edges of the array were set up as zero flux boundaries. Sources of a diffusible morphogen were simulated as short segments that were kept at constant value, either at one of the boundaries, or within the array to produce a trapezoidal internal boundary (Fig. 3). Diffusion was simulated numerically, using a 4th order RungeKutta algorithm (Wilson, 2000). Thresholds were plotted at arbitrary values of the resulting diffusion gradient.

\section{RESULTS}

\section{The wild-type venation and colour pattern}

The normal venation pattern of $P$. xuthus consists of a clearly developed discal cell from which most of the longitudinal veins originate. On the hind wing the wing vein media $3\left(\mathrm{M}_{3}\right)$ is exceptionally long and serves to stabilise the tail, which is typical for the Papilionidae (Fig. 1).

The colour pattern of $P$. xuthus is sexually dimorphic. In the hind wing, the more proximal marginal bands are much broader in the female than in the male, and extend over most of the wing surface (Figs 1b, c). The male colour pattern is therefore more discrete and more suitable for analysis. The normal colour pattern of $P$. xuthus consists primarily of a black pattern on a yellow background. On the hind wing, regions of blue-iridescent and orange scales separate some of the black bands. The black pattern on the dorsal fore wing consists of tapering venous stripes along the basal portions of the longitudinal wing veins, and of a system of nearly parallel marginal bands that run from the anterior to the posterior wing margin. There is one black band close to the wing margin and a pair of paler black bands, also parallel to the wing margin, but further in (Fig. 1b, 2b-c).

Within the discal cell there is a pattern of thin black lines (Fig. 1b, 2b) that reflects the course of the basal branches of the media vein that normally atrophy during the pupal stage to form the discal cell (Behrends, 1936; Kuntze, 1936; Hering, 1940). These patterns are common, particularly in the Papilionidae, and are equivalent to the vein-dependent patterns found elsewhere (Nijhout, 1991). Inside the discal cell there are, in addition, two broad black bands that run perpendicular to the long axis of the cell.

The black bands nearest the wing margin are often scalloped and reveal a row of half-moon-like yellow patches of yellow background colouration along the outer margin, in the middle of each wing-cell. The submarginal bands on the fore wing are slightly dislocated at several locations where they cross a wing vein. On the hind wing, by contrast, the black banding pattern is highly dislocated. Here the black bands are broken up into short segments that run approximately perpendicular to the wing veins, rather than parallel to the wing margin (Fig. 2 a-c).

\section{The mutant venation and colour pattern}

The venation pattern of the veins-reduced mutant is dramatically reduced. In the fore wing, seven of the longitudinal veins are missing, so the venation consists only of the costa, the subcosta, the veins forming a discal cell, and the anal vein. In the hind wing, the subcosta, the discal cell veins and the media 3 are present, and five of the longitudinal veins are missing (Fig. 1d). Thus, most of the veins that, in the wild type, run from the discal cell to the wing margin are missing in the mutant (Fig. 1a, d). Finally, the contour of the distal hind wing margin is different in the mutant. The wild type margin undulates, extending at the wing veins and scalloped between the veins. In the mutants, by contrast, the distal margin is smooth (as indicated by 4 versus $4^{*}$ in Fig. $2 \mathrm{a}, \mathrm{d}$ ).

The pattern differences between wild type and mutant are best visible on the ventral wing surface of the males (Fig. 2). In the mutant the venous stripes are absent where the corresponding veins are missing (Fig. 1e, f; and indicated by $1 / 1 *$ in Fig. 2). In the absence of veins, the black bands are no longer dislocated but now run nearly perfectly parallel to the wing margin. This change in the pattern is particularly striking on the hind wing. Here the

Fig. 1. Wild type venation pattern, (a) and dorsal colour pattern of male (b) and female (c) swallowtail Papilio xuthus. Below corresponding aspects of the veins-reduced mutant are showing the venation pattern (d) and male (e) and female (f) dorsal colour pattern $. \mathrm{D}=$ discal cell, $\mathrm{M}_{3}=$ media 3 .

Fig. 2. The ventral colour pattern of the wild type male ( $a, b)$ differs from the mutant colour pattern ( $d, e)$. In panel (a) only those pattern elements of the wild-type are depicted schematically which are absent or changed in position compared to the mutant pattern (d). Venous stripes which are present in the wild type (a,b) and are missing in the mutant $(\mathrm{d}, \mathrm{e})$ are spotted and indicated by 1 versus $1 *$. Distal parts of the parafocal elements and marginal bands which are dislocated in the wild type (a-c) but are fused to connected bands in the mutant $(\mathrm{d}-\mathrm{f})$ are combed $\left(2 / 2^{*}\right)$ and striped $\left(3 / 3^{*}\right)$ respectively. The lateral hind wing margin of the wild type shows a waved contour with veins rumning to the tips $(a, 4)$. In contrast, the lateral margin of the mutant is smoothened $\left(c, 4^{*}\right)$. 

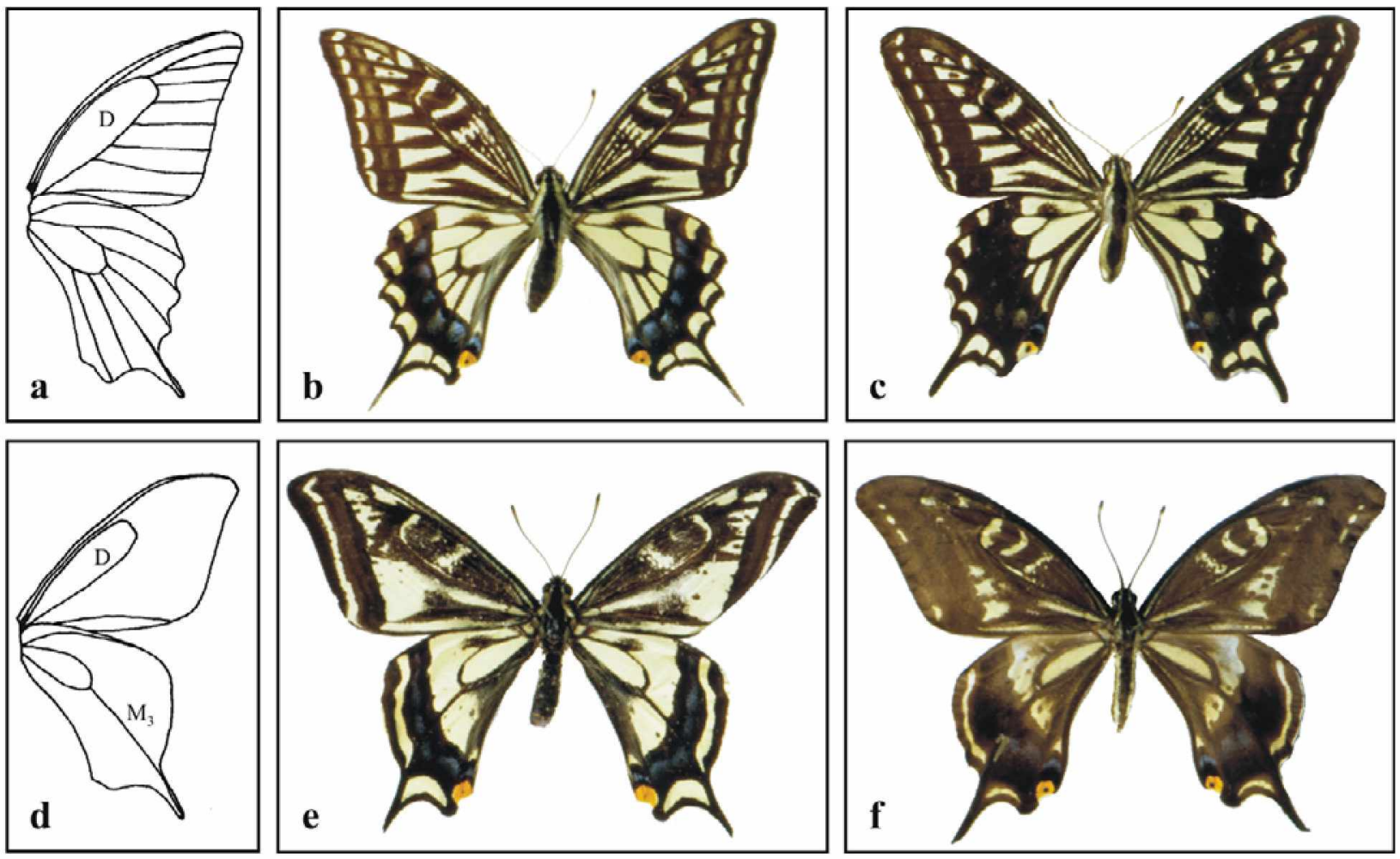

2
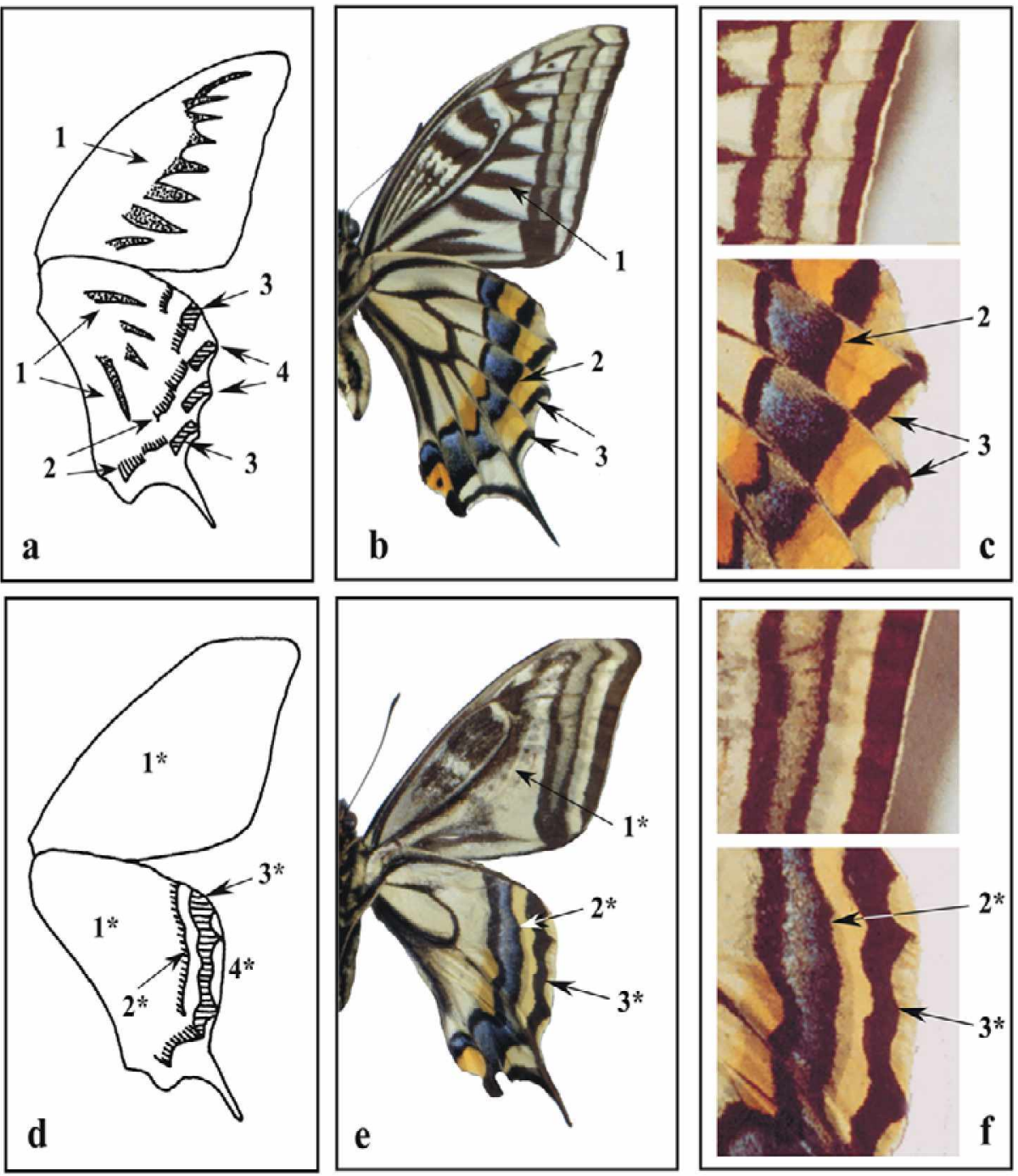


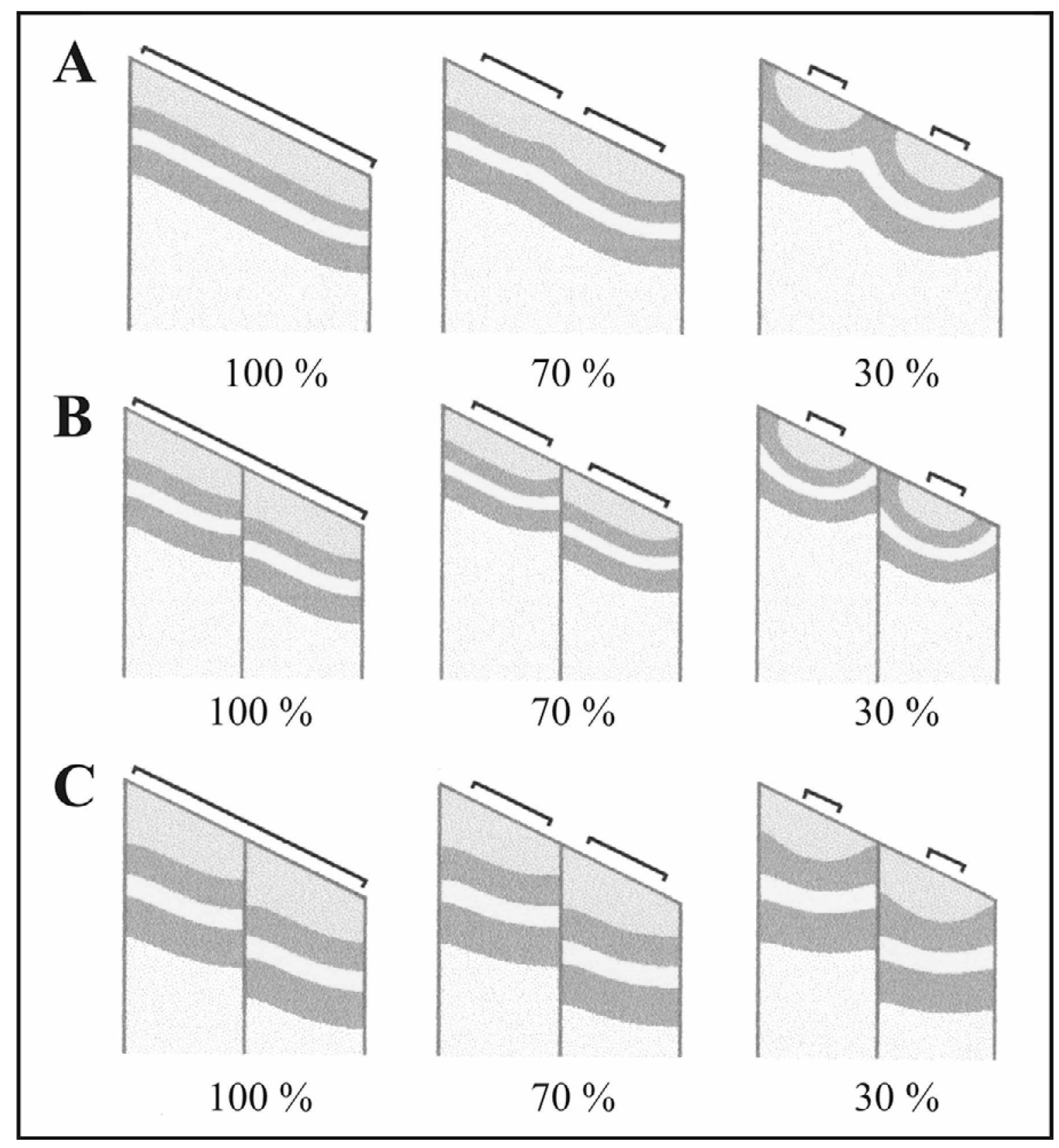

Fig. 3. Computer simulations in a segment of the hind wing two wing-cells wide. Brackets indicate positions of sources, contours are arbitrary, $\mathrm{A}$ - top row = no veins; $\mathrm{B}-$ middle row $=$ with veins as internal no-flux boundary, but short time for diffusion; $\mathrm{C}-$ bottom row $=$ with veins as internal no-flux boundary, and longer time for diffusion. Percentage values indicate relative length of the marginal sources (brackets) as a percentage of the intervein distance.

formerly segmented black bands are now fused and continuous and run almost perfectly parallel to the wing margin (Fig. 2 e-f). The distal-most band is slightly wavy, touching the wing margin in several locations. The orange and iridescent blue patches are also fused in the mutant into continuous bands of colour. The only dislocation occurs at the single remaining longitudinal vein, $\mathrm{M}_{3}$ (Fig. 2 as indicated by $2 / 2^{*}$ and $3 / 3^{*}$ ).

In the veins-reduced mutant of $P$. xuthus the thin black vein-dependent stripes within the discal cell are missing (Fig. 1e-f; Fig. 2e). This observation suggests that the basal portion of the media vein is also missing in the mutant. The fact that the discal cell is complete, however, suggests that the basal portions of the radius and cubitus veins, as well as some of the cross veins that close off the discal cell, did not atrophy. The two broad black cross bands in the discal cell are greatly expanded in the mutant, occupying nearly the entire area of this cell, and their outline is much less distinct than in the wild type. Finally, a fairly broad vein-dependent pattern is developed distal to the veins of the discal cell.

\section{Computer simulations of the effect of veins}

The veins-reduced mutant presents us with a unique opportunity to investigate the potential role of the wing veins in generating the dislocation of the bands of the colour pattern. In particular, we are interested in the 
highly dislocated banding pattern of the hind wing. Here the segments of the band are straight, nearly perpendicular to the veins, and at an angle to the wing margin. In the absence of veins, by contrast, these bands are fused into a single smooth slightly undulating band, parallel to the wing margin. The fact that in the absence of veins the pigment bands are perfectly parallel to the wing margin suggests that the margin acts as an inducing or organising centre for these elements of the colour pattern. This is not an unreasonable supposition. At the time of colour pattern determination there is a lacuna of the circulatory system at the location of the future wing margin (Nijhout, 1991). In addition, several of the genes that are associated with colour pattern induction are initially expressed along the wing margin (Carroll et al., 1994). For purposes of simulation we therefore assume that the wing margin can act as an organising centre for colour pattern formation. The mechanism of pattern induction is unknown. It must involve some kind of signal from the wing margin that spreads proximally into the blade of the wing. In the absence of specific information about how this spread occurs, it is most parsimonious to assume this happens passively by diffusion rather than via a specialised transport mechanism.

We modelled a segment of the hind wing two wingcells wide. Along the middle of the hind wing the veins intersect the margin at an approximately angle of $65^{\circ}$. In our simulations we modelled this by having a sloping internal boundary, represented by the upper margins of the simulation panels in Fig. 3. When we assumed that there were no wing veins and that the upper margin acted as a source of diffusible morphogen, this system produced (as expected) a system of bands parallel to the source, distorted only by the no-flux conditions at the left and right boundaries (Fig. 3A). A simulated vein was then added in the middle of the array in the form of a new no-flux boundary. Figures $3 \mathrm{~B}$ and $3 \mathrm{C}$ illustrate the effect of adding such a vein boundary on the morphology of the banding pattern. Clearly the presence of the vein as a simple boundary causes the banding pattern to become dislocated, even though the source is continuous along the entire margin. But although the bands became dislocated, their morphology and position did not closely resemble that of real bands in the wild type hind wing. In particular, the bands were not perpendicular to the wing veins, as in the real wing.

We simulated other possible properties of wing veins. We simulated veins as constant-level sources of a diffusible substance, and as sinks for the substance diffusing from the wing margin. Neither of these assumptions produced patterns that even remotely resembled those found in normal or veinless specimens.

We observed that in the veinless hind wing the marginal band is not perfectly parallel to the wing margin but undulates slightly. This morphology suggests that the marginal inducing source is probably not continuous. The morphology is consistent with the hypothesis that, in the hind wing at least, the marginal source is broken up into short segments. The positions of the arcs suggests that the presumptive sources must lie at the centre of the presumptive wing cells, that is, roughly half way between the places where wing veins would be expected to meet the wing margin. We simulated this by assuming that the sources of the diffusing chemical occupied $70 \%, 50 \%$ and $30 \%$ of the inter-vein distance along the margin (only the $70 \%$ and $30 \%$ cases are illustrated in Fig. 3). Marginal sources that occupy $70 \%$ and $50 \%$ of the width of a wing cell produce patterns that approximate but not quite resemble those on the normal veined wing. Simulations using marginal sources that occupy $30 \%$ of the width of a wing cell, by contrast, produced bands that were highly dislocated and perpendicular to the wing veins, just like those of the normal wild-type pattern (Figs 2b-c). In the absence of wing veins the $30 \%$ source produced the same kind of undulating pattern that is seen in the veinsreduced wing (for instance the white-coloured band in Fig. 3A $30 \%$ ).

\section{DISCUSSION}

The veins-reduced mutant of $P$. xuthus reported here is the most striking example known to date of a colour pattern aberration that is correlated with a severe deficiency in the venation system. A comparative study of the wild type and veins-reduced wings shows that presence of veins is essential for the normal development of two very different aspects of the colour pattern in P.xuthus. The first of these concerns the vein-dependent patterns. The wedge-shaped venous stripes along the proximal halves of the longitudinal veins are absent when the veins are absent, as are the thin venous stripes within the discal cell. The loss of these two vein-dependent colour pattern elements when veins are lost suggests that the veins somehow control pigment synthesis in their vicinity (Koch et al., 1998). The strong taper of the wedge-shaped venous patterns could be produced in two ways. Either the inductive activity of the veins is not constant along their length, declining distally, or in the distal portion of the wing the surrounding tissue is much less sensitive to the signal emanating from the veins. We have at present no way to distinguish between these alternatives.

The second way in which the veins affect pattern formation is by acting as barriers to inductive signals, effectively compartmentalising portions of the pattern. Our computer simulations show that the very severe pattern dislocation on the hind wing can be completely explained by the simple assumption that the veins act as barriers to signal propagation. In our simulations we used diffusion as the mechanism of signal propagation. We cannot exclude the possibility that a much more complicated signalling mechanism is at work here. Indeed, the morphology of the patterns in Fig. 3 does not depend on the mechanism of signal propagation. Any mechanism in which a signal spreads isotropically in all directions, and where its effects decay with distance, will produce similar patterns. Diffusion merely happens to be the simplest way to accomplish this. The key finding of these simulations is that a purely passive barrier-like function of the wing veins is sufficient to produce the observed dislocated pat- 
tern. In addition, it appears that although on the fore wing the entire wing margin acts as an inductive source, this cannot be the case in the hind wing. The observed patterns of both the wild-type and the veins-reduced phenotypes, can only be produced if the sources at the margin are distributed in small patches. The distribution of these patches is strongly correlated with the wing venation in the wild-type, but evidently does not depend on the wing venation, because these patches persist in the veinsreduced mutant.

It is worth considering how it is possible for a wing vein two have two such apparently disparate functions in pattern formation. The signalling function could be most easily explained by the fact that veins are part of the open circulatory system and they are therefore the only ways in which materials can enter and exit the wing. The haemolymph could thus carry a soluble signal into the wing via the veins from where it diffuses into the developing wing blade. The difficulty with this hypothesis is that it requires veins to be differentially permeable to the signal, so it only diffuses out at certain locations. It is more likely that the epidermal cells around the wing vein can become regionally specialised to produce short-range signalling molecules that can propagate to adjoining cells, either through gap junctions or through the extracellular matrix. Preliminary data on the presence and putative role of gap junctions during the time of pattern formation have been shown in wing discs of Bicyclus anynana (cf. Monteiro, 1996). In the wing imaginal disks of Precis coenia and $B$. anynana, several secreted signalling molecules (such as Distal-less and wingless) are produced along the marginal vein at certain stages in development (Carroll et al., 1994; Brakefield et al., 1996). Indeed, at a certain stage in development the expression of Distal-less becomes restricted to small regions at the midpoints between the wing veins, just as is required to produce the normal pattern in our simulations. The role of wing veins as barriers to signal propagation is most readily accounted for by the localized absence of gap junctions between epidermal cells in the vicinity of the wing veins. If there are no gap junctions then cell-to-cell signalling by molecules dissolved in the cytoplasm would be blocked. Such blockage would produce the no-flux conditions that are necessary to produce the morphologically correct pattern dislocation in our simulations. The disparate functions of veins are thus most likely due to differences in the biological properties of epidermal cells in the vicinity of the wing veins.

ACKNOWLEDGEMENTS. We are very grateful to Dr. $\mathrm{K}$. Endo (Yamaguchi University, Japan) for the opportunity to take photos and for providing mutant specimen of Papilio xuthus. We thank anonymous reviewers for constructive suggestions.

\section{REFERENCES}

Berrends J. 1936: Über die Entwicklung des Lakunen-, Ader-, und Tracheensystems während der Puppenruhe im Flügel der
Mehlmotte Ephestia kühniella Zeller. Zeitschr. Morph. Ökol. Tiere 30: 573-596.

Brakefield P.M. 1998: The evolution-development interface and advances with the eyespot patterns of Bicyclus butterflies. Heredity 80: 265-272.

Brakefield P.M., Gates J., Keys D.N., Kesbeke F., WijngaArden P.J., Monteiro A.F., French V. \& Carroll S.B. 1996: Development, plasticity and evolution of butterfly eyespot patterns. Nature 384: 236-242.

Carroll S.B., Gates J., Keys D.N., Paddock S.W., Panganiban G.E.F., Selegue J.E. \& Williams J.A. 1994: Patter formation and eyespot determination in butterfly wings. Science $\mathbf{2 6 5}$ : 109-114.

French V. \& Brakefield P.M. 1992: The development of eyespot patterns on butterfly wings: Morphogen sources or sinks? Development 116: 103-109.

HERING M. 1940: Lepidopterologisches Wörterbuch. Alfred Kerner Verlag, Stuttgart, 123 pp.

Keys D.N., Lewis D.L., Selegue J.e., Pearson B.J., Goodrich L.V., Johnson R.J., Gates J., Scott M.P. \& Carroll S.B. 1999: Recruitment of a hedgehog regulatory circuit in butterfly eyespot evolution. Science 283: 532-534.

Koch P.B., Keys D.N., Rocheleau Th., Aronstein K., Blackburn M., Carroll S.B. \& Ffrench-Constant R.H. 1998: Regulation of dopadecarboxylase expression during colour pattern formation in wild-type and melanic tiger swallowtail butterflies. Development 125: 2303-2313.

Kuntze H. 1936: Die Flügelentwicklung bei Philosamia cynthia Drury, mit besonderer Berücksichtigung des Geäders, der Lakunen und der Tracheensysteme. Zeitschr. Morph. Ökol. Tiere 30: 544-572.

Monterro A.F. 1996: The Evolutionary Genetics and Developmental Basis of Eyespot Morphology in Butterfly Wings. Dissertation, University of Edinburgh, UK, 119 pp.

Monterro A.F., Brakefield P.M. \& French V. 1997a: Butterfly eyespots: The genetics and development of the colour rings. Evolution 51: 1207-1216.

Monterro A.F., Brakefield P.M. \& French V. 1997b: The genetics and development of an eyespot pattern in the butterfly Bicyclus anynana: response to selection for eyespot shape. Genetics 146: 287-294.

Ninhout H.F. 1980: Pattern formation on lepidopteran wings: determination of an eyespot. Dev.Biol. 80: 267-274.

Nivночт H.F. 1991: The Development and Evolution of Butterfly Wing Patterns. Smithsonian Institution Press, Washington, 297 pp.

NiJHOUT H.F. \& GRUNeRT L.W. 1988: Colour pattern regulation after surgery on the wing discs of Precis coenia (Lepidoptera: Nymphalidae) Development 102: 377-385.

SCHWANWITSCH B.N. 1924: On the groundplan of wing-pattern in nymphalids and certain other families of rhopalocerous Lepidoptera. Proc. Zool. Soc London B 34: 509-528.

SưfFerT F. 1927: Zur vergleichenden Analyse der Schmetterlingszeichnung. Biol.Zentrbl. 47: 385-413.

WILSON W. 2000: Simulating Ecological and Evolutionary Systems in C. Cambridge University Press, Cambridge, 301 pp.

Received July 30, 2001; revised November 5, 2001; accepted November 11, 2001 